\title{
PAPER
}

\section{Specific phobia is a frequent non-motor feature in stiff man syndrome}

\author{
P Henningsen, H-M Meinck
}

J Neurol Neurosurg Psychiatry 2003;74:462-465

\begin{abstract}
Objective: To investigate systematically the rate and type of phobia in stiff man syndrome and its variants, and to compare patients with stiff man syndrome with and without phobia for sociodemographic and neurological characteristics.

Methods: 43 consecutive patients with stiff man syndrome referred to a university department of neurology were assessed using the anxiety disorders interview schedule, revised (ADIS-R), a structured diagnostic interview for anxiety disorders, in addition to a full clinical neurological and psychiatric assessment.

Results: 19 patients (44.2\%) developed task specific phobia-that is, fear and avoidance of situations difficult to master owing to the motor symptoms of stiff man syndrome (such as crossing streets). Three further patients $(7 \%)$ had subthreshold phobia - that is, phobic anxiety without avoidance. There were no significant differences between patients with and without phobia in terms of age, illness duration, type of stiff man syndrome, antibody status, or frequency of falls. Patients with phobia were more likely to present with exaggerated startle responses and to have an initial misdiagnosis of psychogenic movement disorder.

Conclusions: Specific phobia is a frequent non-motor symptom of stiff man syndrome. Early recognition is an important aid to correct diagnosis. The aetiology of phobia in stiff man syndrome is unknown. There is no evidence of a direct pathogenic role of autoantibodies directed against glutamic acid decarboxylase in the development of phobia.
\end{abstract}

Correspondence to: Dr Peter Henningsen, Department of Psychosomatic Medicine, University Hospital, Thibautstr 2, 69115 Heidelberg, Germany; peter_henningsen@ med.uni-heidelberg.de

Received 19 September 2002

In revised form 24

November

Accepted

7 December 2002
S tiff man syndrome is a rare neurological disorder characterised by fluctuating stiffness with superimposed paroxysmal painful spasms of the trunk and legs and absence of neurological signs. ${ }^{2}$ Variants of the disorder include progressive encephalomyelitis with rigidity and myoclonus (PERM), with additional neurological signs such as eye movement disturbance, ataxia, or epilepsy not caused by antispastic drug treatment; and the stiff leg syndrome, where stiffness and spasms are confined to the legs. It is controversial whether stiff man syndrome, stiff leg syndrome, and PERM represent different entities or variable manifestations of the same disease process..$^{3-6} \mathrm{~A}$ high prevalence of autoantibodies against glutamic acid decarboxylase (antiGAD antibodies) in both serum and cerebrospinal fluid,,$^{7-10}$ and the frequent association of stiff man syndrome, stiff leg syndrome, and PERM with other autoimmune disorders such as type 1 diabetes or thyroiditis, ${ }^{67}$ strongly suggest that stiff man syndrome, stiff leg syndrome, and PERM are closely related manifestations of an autoimmune encephalomyelitis. For the sake of simplicity, we use the term stiff man syndrome for this group of disorders and specifically refer to stiff leg syndrome or PERM only if necessary.

Previous unsystematic or anecdotal communications on small numbers of patients with stiff man syndrome reported phobia or phobic anxiety. ${ }^{11-13}$ "Phobia" in this group of patients means excessive fear and avoidance of circumscribed situations which are assumed by patients to be difficult to master because of an increase in stiffness, paroxysmal spasms, or sudden falls. Such situations comprise crossing a street, climbing downstairs without banisters, or walking unaided. Patients may be incapacitated by this form of phobia at least as much as by the motor symptoms themselves. It has been suggested that the presence of this particular anxiety is one of the reasons for the frequent misdiagnosis of psychogenic movement disorder in these patients. ${ }^{112}{ }^{14}$ However, case summaries and reviews of the clinical spectrum of stiff man syndrome often do not mention this seemingly common psychological symptom at all. ${ }^{315}$

Here we report the results of a systematic investigation of anxiety in 43 consecutive patients with stiff man syndrome by applying a structured diagnostic interview, the anxiety disorders interview schedule, revised version. ${ }^{16}{ }^{17}$

\section{METHODS}

\section{Patients}

From 1997 to 2002, all patients with a diagnosis of stiff man syndrome seen at the movement disorders clinic of our department of neurology were offered a multidimensional inpatient diagnostic work up that included neurological, neurophysiological, and psychiatric components. All patients who took part in the inpatient evaluation agreed to take part in all three components.

\section{Neurological assessment}

Neurological assessment comprised physical status, laboratory tests, clinical neurophysiology testing, and, if necessary, magnetic resonance imaging of the brain, spinal cord, or both. Laboratory tests included oral glucose tolerance and HbAlc, serum levels of vitamin B-12 and folic acid, and screening for autoantibodies against glutamic acid decarboxylase, parietal cells, thyreoperoxidase, TSH receptors, and, in cases with a short history, amphiphysin 1 and other antineuronal antibodies. Cerebrospinal fluid was investigated in the majority of patients. Clinical neurophysiology comprised an electromyographic search for continuous motor unit firing in stiff muscles, investigation of exteroceptive spinal (myoclonic reflex spasms) and brain stem reflexes (blink and masseter inhibitory reflexes), and motor, somatosensory, and visual evoked potentials. 


\section{Psychiatric assessment}

Initially, patients took part in a semistructured clinical psychiatric interview in which they were asked about the onset and course of their disorder, and how they were coping with their neurological symptoms and with the process that led to the current diagnosis. In addition, systematic inquiries were made about a past history of depressive and anxiety symptoms and significant life events preceding the onset of motor symptoms. Basic biographical information was also obtained.

The patients then took part in the anxiety disorders section of the ADIS-R (German version). This structured interview has been developed specifically for a diagnostic assessment of anxiety and related mental disorders. It not only allows one to decide whether diagnostic thresholds for particular disorders have been reached, but it also elicits information that is relevant for planning treatment, such as lists of phobically avoided situations. The ADIS-R and its German version have been shown to yield good or excellent test-retest reliability for all anxiety disorders except generalised anxiety disorder $(\kappa$ values of 0.92 and 0.91 , respectively). Both versions have been validated against a whole range of self report questionnaires and other structured interviews. ${ }^{17} 18$

All interviews were done by the same interviewer $(\mathrm{PH})$ who has had extensive training and experience in undertaking clinical and structured interviews.

In view of the fact that patients with disease related stiffness, spasms, and falls may have realistic fears of certain situations, special care was taken to define phobia-in accordance with the diagnostic criteria in the Diagnostic and Statistical Manual (DSM-IV ${ }^{19}$ ) - as fear that clearly exceeds any realistic situational assessment, is seen by the patients themselves as irrational, forms a major component of a patient's sufferings, and leads to clear avoidance behaviour. Three tasks that were known to be relevant as fear inducing from a previous case series ${ }^{11}$ (walking unaided, descending stairs, crossing streets) are not specifically asked for in the structured interview; therefore the appropriate questions were added for the purpose of this study.

\section{RESULTS}

\section{Sample characteristics}

Forty three patients with stiff man syndrome were investigated. The sociodemographic details of the patients are listed in table 1. Most patients had already been treated pharmacologically before the time of interview.

In 19 of the 43 patients, task specific phobia developedthat is, phobic anxiety and avoidance of tasks or situations of sufficient severity to warrant the diagnosis. Three additional patients had the same type of phobic anxiety but without behavioural avoidance, so that only a subthreshold diagnosis of phobia is justified in these cases (table 1).

\section{Clinical description of phobia and phobic anxiety}

No patient had a history of specific phobia independent of stiff man syndrome. In most cases, the phobia developed after the onset of motor symptoms, with a span ranging from no delay (co-occurrence of motor and phobic symptoms) to a delay of two years. In two patients, however, phobic symptoms clearly started several months before the onset of motor symptomsfor example, one patient developed intense fear when climbing a stair six months before stiffness became evident. Clinically, there was a positive correlation between increasing severity of motor symptoms and the development of phobia in individual cases, although, between individuals, the level of severity of motor symptoms at which phobic symptoms developed varied greatly. In 15 of these 19 patients, specific phobia was still present at the time of interview; in four others phobia had resolved parallel to a reduction in motor symptoms accompanying symptomatic treatment.
Table 1 Sociodemographic and neurological details of 43 patients included in the study

\begin{tabular}{ll}
\hline Total number & 43 \\
Male (n (\%)) & $14(32.6)$ \\
Female (n (\%)) & $29(67.4)$ \\
Age (years) & 18 to 75 \\
Range & $52.9(13.8)$ \\
Mean (SD) & \\
Duration of disease (years) & 0.5 to 35 \\
Range & $7.8(7.5)$ \\
Mean (SD) & \\
Type of disease* (n (\%)) & $22(51.2)$ \\
SMS & $15(34.9)$ \\
PERM & $3(7.0)$ \\
SLS & $3(7.0)$ \\
pSMS & \\
Antibody status (n (\%)) & $30(69.8)$ \\
AntiGAD positive & $2(4.7)$ \\
AntiAMP positive & $11(25.6)$ \\
AntiGAD/AMP negative & \\
Task specific phobia (n (\%)) & $19(44.2)$ \\
Present & $3(7.0)$ \\
Phobic anxiety w/o avoidance & $21(48.8)$ \\
Absent &
\end{tabular}

*No patient was diagnosed with SMS and myoclonus (jerking $S M S^{6}$ ). AMP, amphiphysin; GAD, glutamic acid decarboxylase; PERM, progressive encephalomyelitis with rigidity and myoclonus; $\mathrm{PSMS}$, paraneoplastic stiff man syndrome; SLS, stiff leg syndrome; SMS, stiff man syndrome.

The tasks and situations most commonly inducing fear and avoidance were: walking unaided in open space (17), crossing a street (15), and descending (but not climbing) stairs (12). All patients reported fear of at least one of these three specific tasks; most patients reported two or all three. Further questioning often revealed additional fear of common situations and tasks, such as being among people (8), driving in a car (8), being in a closed room (7), and going into shopping centres (7) or restaurants (5). Usually, these more common situations were only avoided when they involved one of the three specific tasks listed above.

The most common fears were as follows: of growing stiff (16), of falling (14), of losing control (12), of ridiculous behaviour (8), of pain (4), of suffocation (2), of going mad (2), and of a heart attack (2).

\section{Comparison of patients with and without phobia}

Twenty one patients with stiff man syndrome did not develop phobia or phobic anxiety. Comparing patients with and without phobia (omitting the three subthreshold cases) did not show significant differences in terms of age, sex, and illness duration (table 2). Most associations between neurological and psychiatric findings were also non-significant. Task specific phobia was evenly distributed among patients with the clinical diagnoses of stiff man syndrome, stiff leg syndrome, and PERM, and among patients with and without antiGAD antibodies (table 2). Moreover, task specific phobia occurred with a similar prevalence among patients with or without a history of falls $(63 \% v 50 \%)$ and among those with or without fractures or subluxations (62\% v 53\%). However, there was a significant association between exaggerated startle reactions and the presence of task specific phobia (table 2 ).

\section{Psychiatric comorbidity, life events, psychogenic} misdiagnosis, and results of psychological treatment Apart from the presence of specific phobia, the psychiatric interview revealed the following pattern of life time psychiatric comorbidity:

- anxiety and/or adjustment disorders in six patients (two with generalised anxiety disorder);

- depression (major depression or dysthymia) in five patients; 
Table 2 Comparison of patients with stiff man syndrome with and without task specific phobias

\begin{tabular}{|c|c|c|c|}
\hline Variable & $\begin{array}{l}\text { Patients with } \\
\text { phobia }\end{array}$ & $\begin{array}{l}\text { Patients without } \\
\text { phobia }\end{array}$ & p Value* \\
\hline Total number & 19 & 21 & \\
\hline Male (n (\%)) & $5(26.3)$ & $7(33.3)$ & \\
\hline Female (n $(\%))$ & 14 (73.7) & $14(66.6)$ & \\
\hline \multicolumn{4}{|l|}{ Age (years) } \\
\hline Mean (SD) & $54.4(14.9)$ & $51.8(13.6)$ & NS \\
\hline \multicolumn{4}{|l|}{ Duration of disease (years) } \\
\hline Mean (SD) & $8.8(7.1)$ & $5.4(5.4)$ & NS \\
\hline \multicolumn{4}{|l|}{ Type of disease (n (\%)) } \\
\hline SMS & $11(57.9)$ & $10(47.6)$ & \\
\hline PERM & $7(36.8)$ & $6(28.6)$ & \\
\hline SLS & $1(5.3)$ & $2(9.5)$ & \\
\hline pSMS & $0(0.0)$ & 3 (14.3) & \\
\hline \multicolumn{4}{|l|}{ Antibody status (n (\%)) } \\
\hline AntiGAD positive & $16(84.2)$ & $13(61.9)$ & \\
\hline AntiAMP positive & $0(0.0)$ & $2(9.5)$ & \\
\hline AntiGAD/AMP negative & $3(15.8)$ & $6(28.6)$ & \\
\hline Exaggerated startle response (n (\%)) & $16(84.2)$ & $11(52.4)$ & $<0.05$ \\
\hline Psychiatric comorbidity present (n $(\%))$ & 11 (57.9) & $8(38.1)$ & NS \\
\hline Significant life event before onset (n (\%)) & $11(57.9)$ & $8(44.4 \dagger)$ & NS \\
\hline Initial psychogenic misdiagnosis (n (\%)) & 15 (78.9) & $8(38.1)$ & $<0.02$ \\
\hline \multicolumn{4}{|c|}{$\begin{array}{l}\text { *Fisher's exact and } t \text { tests, as appropriate. } \\
\text { †Total number for this comparison = } 18 \text { (life events could not be ascertained in three patients). } \\
\text { AMP, amphiphysin; GAD, glutamic acid decarboxylase; PERM, progressive encephalomyelitis with rigidity } \\
\text { and myoclonus; pSMS, paraneoplastic stiff man syndrome; SLS, stiff leg syndrome; SMS, stiff man syndrome }\end{array}$} \\
\hline
\end{tabular}

- personality disorders of varying types in six patients;

- other psychiatric disorders (substance abuse, mild dementia) in four patients.

In 22 patients, no psychiatric comorbidity was detected. Again, comparing the presence or absence of lifetime psychiatric comorbidity in patients with and without specific phobia did not show significant differences, nor did comparison of the rates of important life events before the onset of stiff man syndrome (table 2 ).

In comparison with patients without phobia, in those with phobia there was a significantly higher risk of an initial misdiagnosis of psychogenic movement disorder as a cause for their motor symptoms (table 2).

Psychiatric, psychotherapeutic, and behavioural treatment initiated in seven patients following a misdiagnosis of psychogenic movement disorder was never effective for either non-motor or motor symptoms. In four patients, psychotherapeutic treatments were initiated following a correct diagnosis of stiff man syndrome, specifically for the phobic symptoms. In these patients, there was no demonstrable effect of exposure therapy and other therapeutic techniques on the phobic or motor symptoms. However, as in other patients with stiff man syndrome and phobias, the phobic symptoms decreased or even disappeared when adequate control of the motor symptoms was obtained by pharmacological means.

\section{DISCUSSION}

On the basis of a structured diagnostic interview, our study confirms and extends previous unsystematic observations of a high prevalence of task specific phobias in patients with stiff man syndrome. We found that $44 \%$ of a consecutive sample of 43 patients with stiff man syndrome developed this particular type of phobia, and another 7\% developed subthreshold phobia (anxiety without avoidance). In no patient did a specific phobia occur independently of stiff man syndrome, but in two patients, phobic symptoms developed before the onset of motor symptoms. Patients with stiff man syndrome and specific phobia did not differ significantly from those without phobia in terms of age, sex, disease duration, or psychiatric comorbidity. More importantly, there was no consistent relation between the development of phobia and the clinical manifestation as stiff man syndrome, stiff leg syndrome, or PERM, nor with the presence or absence of antiGAD antibodies. The study confirms earlier suggestions that the presence of phobia increases the risk of a misdiagnosis of psychogenic movement disorder.

The rate of specific phobia in patients with stiff man syndrome is at least five times greater than in the general population: in the national comorbidity survey, the rate of simple phobia (a synonym for specific phobia) was $11.3 \%$ lifetime and 5.5\% current. ${ }^{20}$ Although psychiatric symptoms and disorders are common in neurological disorders, ${ }^{21}$ a rate of about $50 \%$ for one specific form of anxiety disorder without particularly raised rates for depression, other anxiety, or other mental disorders has to be considered very high. Dalakas et al mention the same rate of "task specific phobias" in 10 of 20 patients with stiff man syndrome. ${ }^{12}$

Nosologically, the specificity of these phobias points to a classification of "specific phobia, situational subtype" rather than agoraphobia. ${ }^{1922}$ The term "task specific phobia," which has already been used to describe the fears of patients with stiff man syndrome, ${ }^{12}$ seems to capture well the basic fear underlying this situational type of specificity. In no patient did anxiety and avoidance behaviour become generalised in a way that would justify a diagnosis of agoraphobia (without panic disorder), even later in the course of the illness. On the other hand, the phobia in our patients usually did not involve only the fear of further falling or the fear of free space. Thus subspecification of the specific phobia as "space phobia" ${ }^{23}$ is not appropriate.

The occurrence of this particular anxiety disorder may be a symptom of, or a reaction to, the neurological disorder, a side effect of drug treatment, or a comorbid condition. ${ }^{24}$ Arguing against a merely reactive origin, task specific phobia was distributed fairly evenly among patients with and without gait disturbance or a history of falls, fractures, or subluxations.

Three arguments suggest that the phobic symptoms are a primary manifestation of the disease itself. First, in some patients the phobic symptoms occur before the onset of motor symptoms (this aspect was also described in an earlier case series $\left.{ }^{11}\right)$. Second, there are close temporal correlations between the onset of and decreases in motor and phobic symptoms. Third, phobic cognition remains specific in a 
reliable and stereotypical way, closely related to situations that may be difficult to master because of spasms, stiffness, or falls, and without generalisation to other situations or to specific fears that are most common in the general population, such as fear of animals, height, or flying..$^{25}$ Nevertheless, the results of our study do not rule out the possibility that the specific phobia is a psychological reaction to the motor symptoms, as even in the few cases with phobic symptoms before the onset of obvious motor symptoms, the latter might have been present in a subtle way unacknowledged consciously by the patient.

The pathogenesis of phobic anxiety in patients with stiff man syndrome remains unknown. Recent hypotheses explain human phobic anxiety through vestibular dysfunction ${ }^{2627}$ or discuss a possible role of glutamatergic systems. ${ }^{28}$ With the vestibular hypothesis, one would expect a predominance of patients with clinical or electrophysiological signs of brain stem dysfunction - that is, with PERM - in the phobia group, which was not the case. In patients with stiff man syndrome it is tempting to speculate that central nervous system actions of antiGAD antibodies might be involved, for example by reducing the synthesis of $\mathrm{GABA}^{8}$ the main inhibitory neurotransmitter in the CNS. For instance, mice that are genetically deficient in GAD show increased anxiety-like responses. ${ }^{29}$ However, our results do not suggest an association of stiff man syndrome and phobia with neurotransmitter mechanisms, as there was no consistent correlation between the presence or absence of antiGAD antibodies and the presence of phobia. Five of 13 patients without raised antibody levels also developed phobia or phobic anxiety.

Psychological mechanisms of associative (conditioned) or non-associative learning, or both, will most probably contribute to the development of specific phobia in patients with stiff man syndrome, but the precise nature and interactions of these psychological mechanisms with neurobiologically altered thresholds for fear responses remain to be determined. ${ }^{30}$ The significant association of phobia in stiff man syndrome with exaggerated startle responses might be a point of departure for future research on these interactions.

Limitations of our study concern the fact that we did not test the inter-rater reliability of the diagnoses obtained in the structured interview specifically for our sample. However, the good or even excellent reliability for the diagnoses in question obtained with the ADIS- $\mathrm{R}^{17} 18$ suggests that inter-rater reliability was not a limiting factor in our findings. A second limitation concerns the lack of a control group of patients with similar movement disorders. Without such a control group it is difficult to rule out the possibility that specific phobia in patients with stiff man syndrome is a psychological reaction to unforeseeable spasms and falls. However, apart from the difficulty in defining and arranging for an adequate control group (though patients with the rare disorder of acquired hyperekplexia might be suitable), the main message of this study - that specific phobia is a frequent non-motor feature of stiff man syndrome-is quite independent of whether it also occurs in other neurological disorders.

\section{Conclusions}

Recognition of specific phobia as a common psychological symptom in stiff man syndrome turns it into a truly neuropsychiatric condition. Clinically, the presence of task specific phobia might be a help in the prompt diagnosis of stiff man syndrome, contrary to the widely held belief that marked anxiety is a particular feature of psychogenic movement disorders. Patients with phobias without panic disorder tend not to seek help for their disorder in spite of significant disability, possibly because of embarassment. ${ }^{25}{ }^{30}$ Thus early recognition of phobia in patients with stiff man syndrome is particularly important for devising treatment strategies to help them cope with this aspect of their disease.

\section{ACKNOWLEDGEMENT}

This study was supported by a grant from the Volkswagenstiftung (I / 71452).

\section{Authors' affiliations}

P Henningsen, Department of Psychosomatic Medicine, University

Hospital, Heidelberg, Germany

H-M Meinck, Department of Neurology, University Hospital, Heidelberg

Competing interests: none declared

\section{REFERENCES}

1 Moersch FP, Woltman HW. Progressive fluctuating muscular rigidity and spasm (stiff-man syndrome): report of a case and some observations in 13 other cases. Mayo Clin Proc 1956;31:421-7.

2 Lorish TR, Thorsteinsson G, Howard FM. Stiff-man syndrome updated. Mayo Clin Proc 1989;64:629-36.

3 Brown P, Marsden CD. The stiff man and stiff man plus syndromes. J Neurol 1999;246:648-52.

4 Barker RA, Revesz T, Thom M, et al. Review of 23 patients affected by the stiff man syndrome: clinical subdivision into stiff trunk (man) syndrome, stiff limb syndrome, and progressive encephalomyelitis with rigidity. J Neurol Neurosurg Psychiatry 1998;65:633-40.

5 Saiz A, Graus F, Valldeoriola F, et al. Stiff-leg syndrome: a focal form of stiff-man syndrome. Ann Neurol 1998;43:400-3.

6 Meinck HM, Thompson PD. Stiff man syndrome and related conditions. Mov Disord 2002; 17:853-66.

7 Solimena M, Folli F, Aparisi R, et al. Auto-antibodies to GABAergic neurons and pancreatic beta cells in stiff-man syndrome. N Engl J Med 1990;322: 1555-60.

8 Dinkel K, Meinck HM, Jury KM, et al. Inhibition of gamma-aminobutyric acid synthesis by glutamic acid de-carboxylase autoantibodies in stiff man syndrome. Ann Neurol 1998;44:194-201.

9 Koerner C, Dinkel K, Richter W, et al. Intrathecal synthesis of GAD autoantibodies in stiff man syndrome [abstract]. Clin Neurophysiol 1999;110(suppl 1):S235

10 Dalakas MC, Li M, Fujii M, et al. Stiff person syndrome: quantification, specificity and intrathecal synthesis of GAD65 antibodies. Neurology $2001 ; 57: 780-4$.

11 Henningsen P, Clement U, Küchenhoff J, et al. Psychological factors in the diagnosis and pathogenesis of stiff-man syndrome. Neurology 1996;47:38-42.

12 Dalakas MC, Fujii M, Li M, et al. The clinical spectrum of anti-GAD antibody-positive patients with stiff-person syndrome. Neurology 2000;55:1531-5.

13 Tinsley JA, Barth EM, Black JL, et al. Psychiatric consultations in stiff-man syndrome. J Clin Psychiatry 1997;58:444-9.

14 Kiriakos CR, Franco KN. Stiff-man syndrome: a case report and review of the literature. Psychosomatics 2002;43:243-4.

15 Shaw PJ. Stiff-man syndrome and its variants. Lancet 1999;353:86-7.

16 Di Nardo PA, Barlow DH. Anxiety disorders interview schedule - revised (ADIS-R) [unpublished manuscript]. Albany, NY: State University of New York, 1988.

17 Margraf J, Schneider S, Ehlers A, eds. DIPS. Diagnostisches Interview bei psychischen Störungen. Berlin: Springer-Verlag, 1994.

18 Di Nardo P, Moras K, Barlow DH, et al. Reliability of DSM-III-R anxiety disorder categories. Using the anxiety disorders interview schedule-revised (ADIS-R). Arch Gen Psychiatry 1993;50:251-6.

19 APA. Diagnostic and statistical manual of mental disorders, 4th ed.(DSM-IV). Washington: American Psychiatric Association, 1994.

20 Magee WJ, Eaton WW, Wittchen HU, et al. Agoraphobia, simple phobia, and social phobia in the National Comorbidity Survey. Arch Gen Psychiatry 1996;53:159-68.

21 Lamberg L. Psychiatric symptoms common in neurological disorders. JAMA 2001;286:154-6.

22 Wittchen HU, Reed V, Kessler RC. The relationship of agoraphobia and panic in a community sample of adolescents and young adults. Arch Gen Psychiatry 1998;55:1017-24

23 Marks I. Space "phobia": a pseudo-agoraphobic syndrome. J Neurol Neurosurg Psychiatry 1981;44:387-91.

24 Davies RD, Gabbert SL, Riggs PD. Anxiety disorders in neurologic illness. Curr Treat Options Neurol 2001;3:333-46.

25 Curtis GC, Magee WJ, Eaton WW, et al. Specific fears and phobias. Epidemiology and classification. Br J Psychiatry 1998;173:212-17.

26 Jacob RG, Whitney SL, Detweiler-Shostak G, et al. Vestibular rehabilitation for patients with agoraphobia and vestibular dysfunction: a pilot study. J Anxiety Disord 2001;15:131-46.

27 Jacob RG, Furman JM. Psychiatric consequences of vestibular dysfunction. Curr Opin Neurol 2001;14:41-6.

28 Mathew SJ, Coplan JD, Gorman JM. Neurobiological mechanisms of social anxiety disorder. Am J Psychiatry 2001;158:1558-67.

29 Kash SF, Tecott LH, Hodge C, et al. Increased anxiety and altered responses to anxiolytics in mice deficient in the 65-kDa isoform of glutamic acid decarboxylase. Proc Natl Acad Sci USA 1999;96:1698-703.

30 Fyer AJ. Current approaches to etiology and pathophysiology of specific phobia. Biol Psychiatry 1998;44:1295-304. 